\title{
FACTORING OPERATORS SATISFYING $p$-ESTIMATES
}

\author{
STAN BYRD
}

\begin{abstract}
Necessary and sufficient conditions for a positive operator to factor through a Banach lattice satisfying upper and lower estimates are presented. These conditions are then combined to give a necessary condition for a positive operator to factor through a super-reflexive Banach lattice. An example is given to show that, in spite of the name given by Beauzamy, uniformly convexifying operators need not factor through any uniformly convex lattice
\end{abstract}

0. Introduction. In contrast to the elegant equivalence between super-reflexive Banach spaces and spaces that can be renormed with an equivalent uniformly convex norm, worked out by James and Enflo, the results on operators is not as satisfying or complete. Beauzamy [1] restated some of James' definitions for super-reflexity in terms of operators. The operators he defined to be uniformly convexifying, however, may not even factor through any uniformly convex space. Possibly a better name would be super-reflexive maps since any operator that is finitely representable in a uniformly convexifying operator cannot have the Finite Tree Property. Davis, Figiel, Johnson, and Pełczyński [4] have shown that weak compact maps are "reflexive" in the sense that they factor through reflexive spaces. However, there are no known conditions on $T: X \rightarrow Y$ which are equivalent to $T$ being factorable through a uniformly convex space. In this paper, necessary and sufficient conditions are obtained if $X$ and $Y$ are Banach lattices, $T$ positive, and the factorization uses positive operators.

1. Preliminaries. In this section definitions and results that will be used throughout this paper will be given. Notation will generally be consistent with Lindenstrauss and Tzafriri [9].

A partially ordered normed space $X$ over the reals is called a normed lattice provided

(i) $x \leq y$ implies $x+z \leq y+z$, for every $x, y, z \in X$,

(ii) $a x \geq 0$, for every $x \geq 0$ in $X$ and every real $a \geq 0$,

(iii) for all $x, y \in X$ there exists a least upper bound (lub) $x \vee y$ in $X$, and

(iv) $\|x\| \leq\|y\|$ whenever $|x| \leq|y|$, where the absolute value of $x \in X$, denoted $|x|$, is defined by $|x|=x \vee(-x)$.

If $X$ is a Banach space and also satisfies (i)-(iv), $X$ is called a Banach lattice. The greatest lower bound (glb) of $x$ and $y$ in $X$ will be denoted by $x \wedge y$. If $x \in X$, $x^{+}=x \vee 0$ is called the positive part of $x$ and $x^{-}=-(x \wedge 0)$ is called the negative part of $x$. The set $C=\{x \in X: x \geq 0\}$ is called the positive cone of $X$. It is easy to see that the positive cone completely determines the ordering. A set $\left\{x_{i}\right\}_{i \in \Lambda}$ in

Received by the editors June 1, 1987 .

1980 Mathematics Subject Classification (1985 Revision). Primary 47A68, 46B30.

Key words and phrases. Banach lattice, positive operator, upper and lower estimates. 
$X$ is said to be pariwise disjoint (pwd) if $\left|x_{i}\right| \wedge\left|x_{i}\right|=0$ for $i \neq j$. A linear operator $T: X \rightarrow Y$ where $X$ and $Y$ are normed lattices is said to be a positive operator if $T x \geq 0$ for every $x \geq 0$ in $X$.

Every Banach lattice $X$ has the decomposition property which states that if $x_{1}, x_{2}$ and $y$ are positive elements of $X$ and $y \leq x_{1}+x_{2}$ then there are $y_{1}$ and $y_{2}$ in $X$ with $0 \leq y_{1} \leq x_{1}$ and $0 \leq y_{2} \leq x_{2}$ such that $y=y_{1}+y_{2}$. This can easily be seen by taking $y=x_{1} \wedge y$ and $y_{2}=y-y_{1}$.

A sublattice of a Banach lattice $X$ is a closed linear subspace $Y$ of $X$ so that $Y$ is closed under the lattice operations. An ideal in $X$ is a linear sublattice $Y$ for which $y \in Y$ whenever $|y| \leq|x|$ for some $x \in Y$. If $Y$ is an ideal in $X$ then the quotient space $X / Y$ becomes a Banach lattice if we take as its positive cone the image of the positive cone of $X$.

In a Banach lattice $X$ if $\left\{x_{i}\right\}_{i=1}^{n} \subseteq X$ and $\frac{1}{p}+\frac{1}{q}=1$, the vector

$$
\left(\sum_{i=1}^{n}\left|x_{i}\right|^{p}\right)^{1 / p}=\operatorname{lub}\left\{\sum_{i=1}^{n} a_{i} x_{i}\right\}
$$

in $X$ over all $\left\{a_{i}\right\}_{i=1}^{n}$ in $R^{n}$ for which $\sum_{i=1}^{n}\left|a_{i}\right|^{q} \leq 1$. This lub will always exist in $X$. For a discussion of this see [10, p. 42]. A Banach lattice $X$ is said to be $\sigma$-complete if every order bounded sequence in $X$ has a lub. An ideal $Y$ in a Banach lattice $X$ is called a band if, for every subset $\left\{y_{i}\right\}_{i \in \Lambda}$ of $Y$ such that $\bigvee_{i \in \Lambda} y_{i}$ exists in $X$, this element already belongs to $Y$. A band $Y$ of a Banach lattice $X$ is called a projection band if $X=Y \oplus Y^{\perp}$, where $Y^{\perp}=\{x \in X:|x| \wedge|y|=0$ whenever $y \in Y\}$.

The following lemma of A. I. Yudin which was proved in the more general setting of Archimedean Riesz spaces will be used implicitly throughout this paper.

LEMMA 1.2. Suppose $u$ and $v$ are functions of a finite number of arguments $x_{1}, x_{2}, \ldots, x_{N} \in X$, composed from these arguments with the aid of a finite number of operations of addition, multiplication by real numbers and passage to the supremum. If $u=v$ is true in the special case where $X$ is the reals, then $u=v$ is true in any Banach lattice $X$.

PROOF. [15, p. 145].

LEMMA 1.3. The norm completion of a normed lattice is a Banach lattice.

ProOF. [5, p. 130].

DEFINITION 1.4. For $1<p<\infty$, a Banach lattice $X$ is said to satisfy an upper, respectively, lower p-estimate if there exists a constant $M>0$ such that for every choice of $\left\{x_{i}\right\}_{i=1}^{n}$ pwd in $X$, we have

$$
\left\|\sum_{i=1}^{n} x_{i}\right\| \leq M\left[\sum_{i=1}^{n}\left\|x_{i}\right\|^{p}\right]^{1 / p},
$$

respectively,

$$
\left\|\sum_{i=1}^{n} x_{i}\right\| \geq M^{-1}\left[\sum_{i=1}^{n}\left\|x_{i}\right\|^{p}\right]^{1 / p} .
$$


The smallest possible value of $M$ is called the upper, respectively, lower $p$-estimate constant of $X$.

Sometimes it is preferable to express the existence of an upper or lower $p$-estimate without the explicit use of disjoint vectors.

PROPOSITION 1.5. A Banach lattice $X$ satisfies an upper, respectively, lower p-estimate if and only if

$$
\left\|\bigvee_{i=1}^{n}\left|x_{i}\right|\right\| \leq M\left[\sum_{i=1}^{n}\left\|x_{i}\right\|^{p}\right]^{1 / p},
$$

respectively,

$$
\left\|\sum_{i=1}^{n}\left|x_{i}\right|\right\| \geq M^{-1}\left[\sum_{i=1}^{n}\left\|x_{i}\right\|^{p}\right]^{1 / p}
$$

holds for every choice of $\left\{x_{i}\right\}_{i=1}^{n}$ in $X$.

ProOF. [10, p. 84].

PROPOSITION 1.6. If $X$ is a normed lattice that satisfies an upper, respectively, lower p-estimate then the norm completion of $X, \bar{X}$, satisfies an upper, respectively, lower p-estimate with the same estimate constant.

ProOF. Suppose $X$ satisfies an upper $p$-estimate with constant $M$. Let $\varepsilon>0$ be given and let $\left\{\bar{x}_{i}\right\}_{i=1}^{n}$ be in $\bar{X}$. Choose $\left\{x_{i}\right\}_{i=1}^{n}$ in $X$ so that $\left\|x_{i}-\bar{x}_{i}\right\| \leq \varepsilon / 2^{i / p}$. Then using Proposition 1.5

$$
\begin{aligned}
\left\|\bigvee_{i=1}^{n}\left|\bar{x}_{i}\right|\right\| \leq & \left\|\bigvee_{i=1}^{n}\left|x_{i}-\bar{x}_{i}\right|\right\|+\left\|\bigvee_{i=1}^{n}\left|x_{i}\right|\right\| \\
& \leq \sum_{i=1}^{n}\left\|x_{i}-\bar{x}_{i}\right\|+M\left[\sum_{i=1}^{n}\left\|x_{i}\right\|^{p}\right]^{1 / p} \\
& \leq \frac{2^{1 / p} \varepsilon}{2^{1 / p}-1}+M\left[\sum_{i=1}^{n}\left\|x_{i}-\bar{x}_{i}\right\|^{p}\right]^{1 / p} \\
& +M\left[\sum_{i=1}^{n}\left\|\bar{x}_{i}\right\|^{p}\right]^{1 / p} \\
& \leq \frac{2^{1 / p} \varepsilon}{2^{1 / p}-1}+M \varepsilon+M\left[\sum_{i=1}^{n}\left\|\bar{x}_{i}\right\|^{p}\right]^{1 / p} .
\end{aligned}
$$

Since $\varepsilon$ was arbitrary, $\bar{X}$ satisfies an upper $p$-estimate with estimate constant $M$.

Now suppose $X$ satisfies a lower $p$-estimate with estimate constant $M$. Let $\varepsilon>0$ be given and let $\left\{\bar{x}_{i}\right\}_{i=1}^{n}$ be in $\bar{X}$. Again choose $\left\{x_{i}\right\}_{i=1}^{n}$ in $X$ so that 
$\left\|x_{i}-\bar{x}_{i}\right\| \leq \varepsilon / 2^{i / p}$. Then using Proposition 1.5

$$
\begin{aligned}
\left\|\sum_{i=1}^{n}\left|\bar{x}_{i}\right|\right\| & =\left\|\sum_{i=1}^{n}\left|x_{i}+\bar{x}_{i}-x_{i}\right|\right\| \\
& \geq\left\|\sum_{i=1}^{n}\left|x_{i}\right|\right\|-\left\|\sum_{i=1}^{n}\left|x_{i}-\bar{x}_{i}\right|\right\| \\
& \geq M^{-1}\left[\sum_{i=1}^{n}\left\|x_{i}\right\|^{p}\right]^{1 / p}-\frac{2^{1 / p} \varepsilon}{2^{1 / p}-1} \\
& \geq M^{-1}\left[\sum_{i=1}^{n}\left\|\bar{x}_{i}\right\|^{p}\right]^{1 / p}-M^{-1}\left[\sum_{i=1}^{n}\left\|x_{i}-\bar{x}_{i}\right\|^{p}\right]^{1 / p}-\frac{2^{1 / p} \varepsilon}{2^{1 / p}-1} \\
& \geq M^{-1}\left[\sum_{i=1}^{n}\left\|\bar{x}_{i}\right\|^{p}\right]^{1 / p}-M^{-1} \varepsilon-\frac{2^{1 / p} \varepsilon}{2^{1 / p}-1} .
\end{aligned}
$$

Since $\varepsilon$ is arbitrary, $\bar{X}$ satisfies a lower $p$-estimate with estimate constant $M$.

DEFINITION 1.7. Let $X$ and $Y$ be two Banach spaces. We shall say that $Y$ is finitely representable in $X$ if for every $\varepsilon>0$ and every finite-dimensional subspace $Y^{\circ}$ of $Y$, there exists a finite-dimensional subspace $X^{\circ}$ of $X$ with $\operatorname{dim} Y^{\circ}=\operatorname{dim} X^{\circ}$ and an isomorphism $T$ from $Y^{\circ}$ onto $X^{\circ}$, with $\|T\| \cdot\left\|T^{-1}\right\| \leq 1+\varepsilon$. A Banach space (lattice) $X$ is said to be super-reflexive if every Banach space (lattice) $Y$ that is finitely representable in $X$ is reflexive. A Banach lattice is super-reflexive if and only if there are $p$ and $q$ with $1<p<2<q<\infty$ so that $X$ satisfies an upper $p$-estimate and a lower $q$-estimate. All super-reflexive Banach spaces (lattices) have an equivalent uniformly convex norm (lattice norm). For proofs of these facts see $[10$, p. $59-101]$.

For $1 \leq p<\infty, l_{p}$ will denote the usual Banach space of $p$-summable sequences and $c_{0}$ will denote the Banach space of null sequences.

2. The main theorems. In this section some results on the problem of when an operator $T: X \rightarrow Y$ factors through a uniformly convex Banach space are presented. We will be concerned with the special case when $X$ and $Y$ are Banach lattices and $T$ is a positive operator. Specifically, necessary and sufficient conditions will be established for a positive operator to be factorable through a Banach lattice satisfying either an upper or lower estimate.

THEOREM 2.1. Let $X$ be a Banach lattice and $T: X \rightarrow Y$ be a positive operator for some Banach lattice $Y$. There is a Banach lattice $Z$ which satisfies a lower $q$ estimate and positive operators $R: X \rightarrow Z$ and $S: Z \rightarrow Y$ so that $T=S R$ if and only if there is a $K>0$ so that for all $\left\{x_{i}\right\}_{i=1}^{n}$, pwd in $X$,

$$
\left[\sum_{i=1}^{n}\left\|T x_{i}\right\|_{Y}^{q}\right]^{1 / q} \leq K\|x\|_{X}
$$

for all $x$ in $X$ with $\left|\sum_{i=1}^{n} x_{i}\right| \leq|x|$. 
Proof. (SUfFiCIENCY) Suppose $T=S R$ and $M$ is the lower $q$-estimate constant for $Z$. Then for each $\left\{x_{i}\right\}_{i=1}^{n}$, pwd in $X$,

$$
\begin{aligned}
{\left[\sum_{i=1}^{n}\left\|T x_{i}\right\|_{Y}^{q}\right]^{1 / q} } & =\left[\sum_{i=1}^{n}\left\|S R x_{i}\right\|_{Y}^{q}\right]^{1 / q} \\
& \leq\|S\|\left[\sum_{i=1}^{n}\left\|R x_{i}\right\|_{Z}^{q}\right]^{1 / q} \leq\|S\| M\left\|\sum_{i=1}^{n} R x_{i}\right\|_{Z} \\
& \leq\|S\| M\|R\|\left\|\sum_{i=1}^{n} x_{i}\right\|_{X} \leq\|S\| M\|R\|\|x\|_{X}
\end{aligned}
$$

for all $x$ in $X$ with $\left|\sum_{i=1}^{n} x_{i}\right| \leq|x|$ and the conclusion holds with $K=\|S\| M\|R\|$.

(NECESSITY) Define $\|\cdot\|_{Z}$ on $T(X)$ by

$$
\|T x\|_{Z}=\sup \left\{\left[\sum_{i=1}^{n}\left\|T x_{i}\right\|_{Y}^{q}\right]^{1 / q}\right\},
$$

where the sup is taken over all $\left\{x_{i}\right\}_{i=1}^{n}$ pwd in $X$ with $\left|\sum_{i=1}^{n} x_{i}\right| \leq|x|$. Note that for all $x$ in $X,\|T x\|_{Y} \leq\|T x\|_{Z}$. Let $Z_{0}=\left\{y \in T(X):\|y\|_{Z}<\infty\right\}$, and let $\varepsilon>0$ be given. For $x$ in $X$ choose $\left\{x_{i}\right\}_{i=1}^{n}$ pwd in $X$ with $\left|\sum_{i=1}^{n} x_{i}\right| \leq|x|$ so that $\|T x\|_{Z} \leq\left[\sum_{i=1}^{n}\left\|T x_{i}\right\|_{Y}^{q}\right]^{1 / q}+\varepsilon$. Then

$$
\|T x\|_{Z} \leq K\left\|\sum_{i=1}^{n} x_{i}\right\|_{X}+\varepsilon \leq K\|x\|_{X}+\varepsilon .
$$

Since $\varepsilon$ is arbitrary, the norm of $T: X \rightarrow Z_{0}$ is bounded above by $K$ and $Z_{0}=T(X)$.

By taking the quotient of $X$ with the ideal $\operatorname{Ker} T$, if necessary, we may assume that $\operatorname{Ker} T$ is trivial. It is clear from the definitioin that $\|T x \mid\|_{Z}=\|T x\|_{Z}$ for all $x$ in $X$. To prove that $\|\cdot\|_{z}$ is monotone we may assume $0 \leq T x \leq T y$. Let $\left\{x_{i}\right\}_{i=1}^{n}$ be pwd in $X$ with $\left|\sum_{i=1}^{n} x_{i}\right| \leq x \leq y$. Then $\left[\sum_{i=1}^{n}\left\|T x_{i}\right\|_{Y}^{q}\right]^{1 / q} \leq\|T y\|_{Z}$ and hence $\|T x\|_{Z} \leq\|T y\|_{z}$.

To verify the triangle inequality let $x$ and $y$ be positive in $X$ and let $\varepsilon>0$ be given. Pick $\left\{z_{i}\right\}_{i=1}^{n}$ pwd in $X$ so that $0 \leq z_{i} \leq x+y, \sum_{i=1}^{n} z_{i} \leq x+y$, and $\|T x+T y\|_{z} \leq\left[\sum_{i=1}^{n}\left\|T z_{i}\right\|_{Y}^{q}\right]^{1 / q}+\varepsilon$. Using the decomposition property in $X$, we can find $\left\{x_{i}\right\}_{i=1}^{n}$ and $\left\{y_{i}\right\}_{i=1}^{n}$ both pwd in $X$ so that $z_{i}=x_{i}+y_{i}, 0 \leq x_{i} \leq z_{i}$, $0 \leq y_{i} \leq z_{i}, \sum_{i=1}^{n} x_{i} \leq x$, and $\sum_{i=1}^{n} y_{i} \leq y$. Using the triangle inequality in $l_{q}$ we have

$$
\begin{aligned}
\|T x+T y\|_{Z} & \leq\left[\sum_{i=1}^{n}\left\|T z_{i}\right\|_{Y}^{q}\right]^{1 / q}+\varepsilon \\
& \leq\left[\sum_{i=1}^{n}\left\|T x_{i}\right\|_{Y}^{q}\right]^{1 / q}+\left[\sum_{i=1}^{n}\left\|T y_{i}\right\|_{Y}^{q}\right]^{1 / q}+\varepsilon \\
& \leq\|T x\|_{Z}+\|T y\|_{Z}+\varepsilon .
\end{aligned}
$$

Since $\varepsilon$ is arbitrary $\|T x+T y\|_{Z} \leq\|T x\|_{Z}+\|T y\|_{Z}$. For general $x$ and $y$ in $X$

$$
\begin{aligned}
\|T x+T y\|_{Z} & =\||T x+T y|\|_{Z} \leq\||T x|+|T y|\|_{Z} \\
& \leq\||T x|\|_{Z}+\|\mid T y\|_{Z}=\|T x\|_{Z}+\|T y\|_{z} .
\end{aligned}
$$


$\left(Z_{0},\|\cdot\|_{Z}\right)$ is a normed lattice with the $Y$-ordering. Let $Z$ be the norm completion of $Z_{0}$ with respect to $\|\cdot\|_{Z}$. By Lemma $1.3,\left(Z,\|\cdot\|_{Z}\right)$ is a Banach lattice. Since $\|\cdot\|_{Z}$-convergence implies $\|\cdot\|_{Y}$-convergence, $Z \subseteq Y$.

It will now be shown that $Z$ satisfies a lower $q$-estimate. Let $\varepsilon>0$ be given and let $x$ and $y$ be disjoint in $X$. Pick $\left\{x_{i}\right\}_{i=1}^{n}$ and $\left\{y_{i}\right\}_{i=1}^{n}$ both pwd in $X$ so that $\left|\sum_{i=1}^{n} x_{i}\right| \leq|x|,\left|\sum_{i=1}^{n} y_{i}\right| \leq|y|,\|T x\|_{Z} \leq(1+\varepsilon)\left[\sum_{i=1}^{n}\left\|T x_{i}\right\|_{Y}^{q}\right]^{1 / q}$ and $\|T y\|_{Z} \leq(1+\varepsilon)\left[\sum_{i=1}^{n}\left\|T y_{i}\right\|_{Y}^{q}\right]^{1 / q}$. Since $x$ and $y$ are disjoint, $\left\{x_{i}\right\}_{i=1}^{n} \cup\left\{y_{i}\right\}_{i=1}^{n}$ is pwd in $X$ and

$$
\begin{aligned}
{\left[\|T x\|_{Z}^{q}+\|T y\|_{Z}^{q}\right]^{1 / q} } & \leq(1+\varepsilon)\left[\sum_{i=1}^{n}\left\|T x_{i}\right\|_{Y}^{q}+\sum_{i=1}^{n}\left\|T y_{i}\right\|_{Y}^{q}\right]^{1 / q} \\
& \leq(1+\varepsilon)\|T x+T y\|_{Z} .
\end{aligned}
$$

Since $\varepsilon$ is arbitrary we have

$$
\left[\|T x\|_{Z}^{q}+\|T y\|_{Z}^{q}\right]^{1 / q} \leq\|T x+T y\|_{Z} .
$$

This inequality can be extended by induction to any finite pwd collection in $X$ and so $Z_{0}$ satisfies a lower $q$-estimate with estimate constant of one. By Proposition 1.6, $Z$ also satisfies a lower $q$-estimate with estimate constant of one.

Define $R: X \rightarrow Z$ by $R x=T x$. $T: X \rightarrow Z_{0}$ has been shown to be continuous and so $R$ is continuous and positive. Define $\bar{S}: Z_{0} \rightarrow Y$ by $\bar{S} z=z$. Then $\|\bar{S} z\|_{Y}=$ $\|z\|_{Y} \leq\|z\|_{Z}$ and $\bar{S}$ is continuous and positive. Letting $S$ be the extension of $\bar{S}$ to $Z$, we get the desired operator. Finally, $T=S R$ by construction.

REMARK. An immediate consequence of Theorem 2.1 is that if the positive operator $T$ can be factored using positive operators through a Banach lattice with a lower $q$-estimate, then $T$ can be factored through $Z$ as constructed in the proof. The space $Z$ has the additional property that its lower $q$-estimate constant is one.

THEOREM 2.2. Let $X$ be a Banach lattice and $T: X \rightarrow Y$ be a positive operator for some Banach lattice $Y$. There is a Banach lattice $Z$ that satisfies an upper $p$ estimate and positive operators $R: X \rightarrow Z$ and $S: Z \rightarrow Y$ with $T=S R$ if and only if there is a $K>0$ so that for all $\left\{x_{i}\right\}_{i=1}^{n}$, pwd in $X$,

$$
\left\|\sum_{i=1}^{n} T x_{i}\right\|_{Y} \leq K\left[\sum_{i=1}^{n}\left\|x_{i}\right\|_{X}^{p}\right]^{1 / p} .
$$

Proof. (SUfficiency) Suppose $T=S R$ and $M$ is the upper $p$-estimate constant of $Z$, then

$$
\begin{aligned}
\left\|\sum_{i=1}^{n} T x_{i}\right\|_{Y} & =\left\|\sum_{i=1}^{n} S R x_{i}\right\|_{Y} \leq\|S\|\left\|\sum_{i=1}^{n} R x_{i}\right\|_{Z} \\
& \leq\|S\| M\left[\sum_{i=1}^{n}\left\|R x_{i}\right\|_{Z}^{p}\right]^{1 / p} \\
& \leq\|S\| M\|R\|\left[\sum_{i=1}^{n}\left\|x_{i}\right\|_{X}^{p}\right]^{1 / p}
\end{aligned}
$$

and the conclusion holds with $K=\|S\| M\|R\|$. 
(NECESSITY) By taking the quotient of $X$ with the ideal $\operatorname{Ker} T$, if necessary, we may assume that $\operatorname{Ker} T$ is trivial.

Define $[[\cdot]]$ on $X$ by $[[x]]=\inf \left\{\left[\sum_{i=1}^{n}\left\|x_{i}\right\|_{X}^{p}\right]^{1 / p}\right\}$ where the inf is taken over all $\left\{x_{i}\right\}_{i=1}^{n}$ pwd in $X$ with $\sum_{i=1}^{n} x_{i}=x$. Note that $[[x]]=[[|x|]]$ and for $x \neq 0$, $0<\|T x\|_{Y} \leq K[[x]]$. Also, if $0 \leq x \leq y$ in $X$ and $y=\sum_{i=1}^{n} y_{i},\left\{y_{i}\right\}_{i=1}^{n}$ pwd in $X$, setting $x_{i}=y_{i} \wedge x$, we get that $\left\{x_{i}\right\}_{i=1}^{n}$ is pwd in $X, \sum_{i=1}^{n} x_{i}=\sum_{i=1}^{n}\left(y_{i} \wedge x\right)=$ $x \wedge y=x$ and

$$
\left[\sum_{i=1}^{n}\left\|x_{i}\right\|_{X}^{p}\right]^{1 / p} \leq\left[\sum_{i=1}^{n}\left\|y_{i}\right\|_{X}^{p}\right]^{1 / p}
$$

This implies that $[[x]] \leq[[y]]$. Also note that if $x$ and $y$ are disjoint in $X,[[x+y]] \leq$ $\left[[[x]]^{p}+[[y]]^{p}\right]^{1 / p} \leq[[x]]+[[y]]$.

$[[\cdot]]$ defined on $X$ satisfies all the properties of a lattice norm except possibly the triangle inequality. By convexifying the unit ball of $(X,[[\cdot]])$ we obtain a norm $\|\cdot\|_{Z}$ defined on $X$ by $\|x\|_{z}=\inf \left\{\sum_{i=1}^{n}\left[\left[x_{i}\right]\right]\right\}$ where the inf is taken over all $\left\{x_{i}\right\}_{i=1}^{n}$ in $X$ with $\sum_{i=1}^{n} x_{i}=x$. It remains to be shown that $\|\cdot\|_{z}$ is a lattice norm.

First we prove the useful equation $\|z\|_{z}=\inf \left\{\sum_{i=1}^{n}\left[\left[x_{i}\right]\right]\right\}$ where the inf is taken over all $\left\{x_{i}\right\}_{i=1}^{n}$ in $X$ with $z=\sum_{i=1}^{n} x_{i}, z^{+}=\sum_{i=1}^{n} x_{i}^{+}, z^{-}=\sum_{i=1}^{n} x_{i}^{-}$, and $\left|x_{i}\right| \leq|z|$. Clearly $\|z\|_{z} \leq$ this infimum.

In a special case, if $z=z_{1}+z_{2}$, then $\left(z_{1}+z_{2}\right)^{+} \leq z_{1}^{+}+z_{2}^{+}$. Set $x_{1}^{+}=z^{+} \wedge z_{1}^{+}$ and $x_{2}^{+}=z^{+}-x_{1}^{+}$. Then $x_{1}^{+}+x_{2}^{+}=z^{+}, x_{1}^{+} \leq z_{1}^{+} \leq\left|z_{1}\right|$ and $x_{2}^{+}=z^{+}-x_{1}^{+}=$ $\left.\mid z^{+} \wedge\left(z_{1}^{+}+z_{2}^{+}\right)\right]-x_{1}^{+}=\left(z^{+} \wedge z_{1}^{+}\right)+\left(z^{+} \wedge z_{2}^{+}\right)-x_{1}^{+} \leq x_{1}^{+}+z_{2}^{+}-x_{1}^{+}=z_{2}^{+} \leq\left|z_{2}\right|$. Similarly, we can find $x_{1}^{-}$and $x_{2}^{-}$so that $x_{1}^{-}+x_{2}^{-}=z^{-}, x_{1}^{-} \leq\left|z_{1}\right|$ and $x_{2}^{-} \leq\left|z_{2}\right|$. Set $x_{1}=x_{1}^{+}-x_{1}^{-}$and $x_{2}=x_{2}^{+}-x_{2}^{-}$. If $z=\sum_{i=1}^{n} z_{i}$, the above results can be extended by induction to find $\left\{x_{i}\right\}_{i=1}^{n}$ in $X$ with $\sum_{i=1}^{n} x_{i}=z, \sum_{i=1}^{n} x_{i}^{+}=z^{+}$, $\sum_{i=1}^{n} x_{i}^{-}=z^{-}$and $\left|x_{i}\right| \leq\left|z_{i}\right|$. Then $\sum_{i=1}^{n}\left[\left[x_{i}\right]\right] \leq \sum_{i=1}^{n}\left[\left[z_{i}\right]\right]$ and the reverse inequality is proven.

If $0 \leq|x| \leq|y|$ and $\left\{y_{i}\right\}_{i=1}^{n}$ satisfies $\sum_{i=1}^{n} y_{i}=y, \sum_{i=1}^{n} y_{i}^{+}=y^{+}, \sum_{i=1}^{n} y_{i}^{-}=y^{-}$ and $\left|y_{i}\right| \leq|y|$, setting $x_{i}^{+}=x^{+} \wedge y_{i}^{+}$and $x_{i}^{-}=x^{-} \wedge y_{i}^{-}$we get that $\sum_{i=1}^{n} x_{i}^{+}=$ $x^{+} \wedge y^{+}=x^{+} \leq|x|, \sum_{i=1}^{n} x_{i}^{-}=x^{-} \wedge y^{-}=x^{-} \leq|x|, \sum_{i=1}^{n} x_{i}=x$ and $\left|x_{i}\right| \leq|x|$. Then $\left|x_{i}\right| \leq\left|y_{i}\right|$ which implies $\sum_{i=1}^{n}\left[\left[x_{i}\right]\right] \leq \sum_{i=1}^{n}\left[\left[y_{i}\right]\right]$ or $\|x\|_{z} \leq\|y\|_{z}$.

Let $Z$ be the $\|\cdot\|_{Z}$-completion of $\left(X,\|\cdot\|_{Z}\right)$. By Lemma $1.3, Z$ is a Banach lattice.

To show $\left(X\|\cdot\|_{z}\right)$ satisfies an upper $p$-estimate, let $\varepsilon>0$ be given and let $x$ and $y$ be disjoint in $X$. We may assume, without loss of generality, that $x$ and $y$ are both positive. Let $\left\{x_{i}\right\}_{i=1}^{n}$ and $\left\{y_{i}\right\}_{i=1}^{n}$ be in $X$ so that $x_{i} \geq 0, y_{i} \geq 0, \sum_{i=1}^{n} x_{i}=x$, $\sum_{i=1}^{n} y_{i}=y, \sum_{i=1}^{n}\left[\left[x_{i}\right]\right]=A \leq\|x\|_{z}+\varepsilon$ and $\sum_{i=1}^{n}\left[\left[y_{i}\right]\right]=B \leq\|y\|_{z}+\varepsilon$. Pick $N$ so that there exist collections of nonnegative integers $\left\{p_{i}\right\}_{i=1}^{n}$ and $\left\{q_{i}\right\}_{i=1}^{n}$ with $N=\sum_{i=1}^{n} p_{i}=\sum_{i=1}^{n} q_{i}$, and for each $i,\left|\left[\left[x_{i}\right]\right]-p_{i} A / N\right|<\varepsilon / n$ and $\left|\left[\left[y_{i}\right]\right]-q_{i} B / N\right|<$ $\varepsilon / n$. Let $w_{j}=x_{i} / p_{i}$ for $\sum_{k=1}^{i-1} p_{k}<j \leq \sum_{k=1}^{i} p_{k}$ and $z_{j}=y_{i} / q_{i}$ for $\sum_{k=1}^{i-1} q_{k}<$ $j \leq \sum_{k=1}^{i} q_{k}$. We have then $\left|\left[\left[w_{j}\right]\right]-A / N\right|=\left(1 / p_{i}\right)\left|\left[\left[x_{i}\right]\right]-p_{i} A / N\right|<\varepsilon / p_{i} n$ and $\left|\left[\left[z_{j}\right]\right]-B / N\right|=\left(1 / q_{i}\right)\left|\left[\left[y_{i}\right]\right]-q_{i} B / N\right|<\varepsilon / q_{i} n$ for $\sum_{k=1}^{i-1} p_{k}<j \leq \sum_{k=1}^{i} p_{k}$ and 
$\sum_{k=1}^{i-1} q_{k}<j \leq \sum_{k=1}^{i} q_{k}$, respectively. Since $w_{j} \wedge z_{j} \leq x \wedge y=0$, we have

$$
\begin{aligned}
\|x+y\|_{z} & \leq \sum_{j=1}^{N}\left[\left[w_{j}+z_{j}\right]\right] \leq \sum_{j=1}^{N}\left[\left[\left[w_{j}\right]\right]^{p}+\left[\left[z_{j}\right]\right]^{p}\right]^{1 / p} \\
& =\sum_{j=1}^{N}\left[\left[\frac{A}{N}+\left[\left[w_{j}\right]\right]-\frac{A}{N}\right]^{p}+\left[\frac{B}{N}+\left[\left[z_{j}\right]\right]-\frac{B}{N}\right]^{p}\right]^{1 / p} \\
& \leq\left(A^{p}+B^{p}\right)^{1 / p}+\sum_{j=1}^{N}\left[(\underbrace{\frac{\varepsilon}{p_{i} N}}_{p_{i} \text { terms }})^{p}+(\underbrace{\frac{\varepsilon}{q_{i} N}}_{q_{i} \text { terms }})^{p}\right]^{1 / p} \\
& \leq\left(A^{p}+B^{p}\right)^{1 / p}+\sum_{j=1}^{N}\left[(\underbrace{\frac{\varepsilon}{p_{i} N}}_{p_{i} \text { terms }})+(\underbrace{\frac{\varepsilon}{q_{i} N}}_{q_{i} \text { terms }})\right] \\
& \leq\left[\left(\|x\|_{Z}+\varepsilon\right)^{p}+\left(\|y\|_{Z}+\varepsilon\right)^{p}\right]^{1 / p}+2 \varepsilon .
\end{aligned}
$$

Since $\varepsilon$ is arbitrary we get $\|x+y\|_{Z} \leq\left(\|x\|_{Z}^{p}+\|y\|_{Z}^{p}\right)^{1 / p}$. This result can be extended by induction to any finite pwd collection in $X$ and $\left(X,\|\cdot\|_{Z}\right)$ satisfies an upper $p$-estimate with estimate constant of one. By Proposition 1.6, $\left(Z,\|\cdot\|_{Z}\right)$ also satisfies an upper $p$-estimate with estimate constant of one.

Define $R: X \rightarrow Z$ by $R x=x$. Since $\|R x\|_{Z}=\|x\|_{Z} \leq\|x\|_{X}, R$ is continuous and positive. Define $\bar{S}:\left(X,\|\cdot\|_{Z}\right) \rightarrow Y$ by $\bar{S} x=T x$. Let $\varepsilon>0$ be given and let $x$ be in $X$. Pick $\left\{x_{i}\right\}_{i=1}^{n}$ in $X$ so that $\sum_{i=1}^{n} x_{i}=x$ and $\sum_{i=1}^{n}\left[\left[x_{i}\right]\right] \leq(1+\varepsilon)\|x\|_{z}$. Further, pick $\left\{x_{i j}\right\}_{j=1}^{k}$ pwd in $X$ so that $\sum_{j=1}^{k} x_{i j}=x_{i}$ and $\left[\sum_{j=1}^{k}\left\|x_{i j}\right\|_{X}^{p}\right]^{1 / p} \leq(1+\varepsilon)\left[\left[x_{i}\right]\right]$. Then

$$
\begin{aligned}
\|\bar{S} x\|_{Y} & =\left\|\sum_{i=1}^{n} \sum_{j=1}^{k} T x_{i j}\right\|_{Y} \leq \sum_{i=1}^{n}\left\|\sum_{j=1}^{k} T x_{i j}\right\|_{Y} \\
& \leq K \sum_{i=1}^{n}\left[\sum_{j=1}^{k}\left\|x_{i j}\right\|_{X}^{p}\right]^{1 / p} \\
& \leq(1+\varepsilon) K \sum_{i=1}^{n}\left[\left[x_{i}\right]\right] \leq(1+\varepsilon)^{2} K\|x\|_{Z} .
\end{aligned}
$$

Since $\varepsilon$ is arbitrary we have $\bar{S}$ is continuous and positive with $\|\bar{S}\| \leq K$. Letting $S$ be the extension of $\bar{S}$ on $Z$, we have the desired operator. Finally $T=S R$ by construction.

REMARK. Like before, Theorem 2.2 implies that if a positive operator $T$ can be factored through a lattice with an upper $p$-estimate, then it can be factored through a lattice whose upper $p$-estimate constant is one.

3. Consequences of the main theorems. In this section several direct consequences of the main theorems are given. In particular, a necessary condition is 
established for a positive operator to be factorable through a super-reflexive Banach lattice.

Beauzamy in [1] introduced the notion of a uniformly convexifying operator. As we shall see later, the definition implies that a Banach space $X$ is uniformly convex if and only if the identity operator on $X$ is uniformly convexifying. An example is presented of a positive uniformly convexifying operator that can be factored with positive operators through no uniformly convex Banach lattice.

THEOREM 3.1. Let $1<p<\infty$. A Banach lattice satisfies an upper, respectively, lower $p$-estimate if and only if its dual $X^{*}$ satisfies a lower, respectively, upper q-estimate, where $\frac{1}{p}+\frac{1}{q}=1$.

PROOF [10, p. 83].

COROLlary 3.2. If $X$ is a Banach lattice $T: X \rightarrow Y$ is a positive operator for some Banach lattice $Y$ and there is a $K>0$ so that for all $\left\{x_{i}\right\}_{i=1}^{n}$ pwd in $X$, $\left[\sum_{i=1}^{n}\left\|T x_{i}\right\|_{Y}^{q}\right]^{1 / q} \leq K\|x\|_{X}$ for all $x$ in $X$ with $\left|\sum_{i=1}^{n} x_{i}\right| \leq|x|$, then the adjoint of $T, T^{*}$, can be factored using positive operators through a Banach lattice which satisfies an upper p-estimate, $\frac{1}{p}+\frac{1}{q}=1$.

PROOF. This follows directly from Theorem 3.1, Theorem 2.1, and the fact that the adjoint of a positive operator is positive.

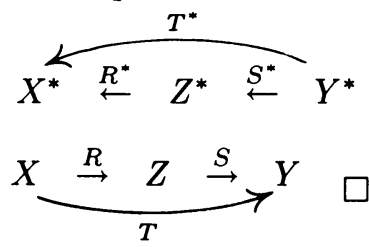

COROLlaRY 3.3. If $X$ is a Banach lattice, $T: X \rightarrow Y$ is a positive operator for some Banach lattice $Y$, and there is a $K>0$ so that for all $\left\{x_{i}\right\}_{i=1}^{n}$ pwd in $X$,

$$
\left\|\sum_{i=1}^{n} T x_{i}\right\|_{Y} \leq K\left[\sum_{i=1}^{n}\left\|x_{i}\right\|_{X}^{p}\right]^{1 / p}
$$

then the adjoint of $T, T^{*}$, can be factored using positive operators through a Banach lattice which satisfies a lower q-estimate, $\frac{1}{p}+\frac{1}{q}=1$.

PROOF. This follows directly from Theorem 3.1 and Theorem 2.2.

COROLlaRY 3.4. Let $X$ be a $\sigma$-complete Banach lattice and $T: X \rightarrow Y$ be a positive operator for some Banach lattice $Y$. There is a Banach lattice $Z$ which satisfies a lower q-estimate and positive operators $R: X \rightarrow Z$ and $S: Z \rightarrow Y$ with $T=S R$ if and only if there is a $K>0$ so that for all $\left\{x_{i}\right\}_{i=1}^{n}$ pwd in $X$

$$
\left[\sum_{i=1}^{n}\left\|T x_{i}\right\|_{Y}^{q}\right]^{1 / q} \leq K\left\|\sum_{i=1}^{n} x_{i}\right\|_{X} .
$$

PROOF. (SUFFICIENCY) This is the special case of Theorem 2.1 with $\sum_{i=1}^{n} x_{i}=$ $x$. 
(NECESSiTY) Define $\|\cdot\|_{Z}$ on $T(X)$ by

$$
\|T x\|_{Z}=\sup \left\{\left[\sum_{i=1}^{n}\left\|T x_{i}\right\|_{Y}^{q}\right]^{1 / q}\right\}
$$

where the sup is taken over all $\left\{x_{i}\right\}_{i=1}^{n}$ pwd in $X$ with $\sum_{i=1}^{n} x_{i}=x$. The proof is essentially the same as the necessity proof in Theorem 2.1 except for the monotonicity of $\|\cdot\|_{z}$. Here, if $0 \leq x \leq y$, then for each $\left\{x_{i}\right\}_{i=1}^{n}$ pwd in $X$ with $x_{i} \geq 0$ and $\sum_{i=1}^{n} x_{i}=x$, setting $y_{i}=\sup _{k}\left\{k x_{i} \wedge y\right\}$ and $y_{0}=y-\sum_{i=1}^{n} y_{i}$, we get that $\left\{y_{i}\right\}_{i=0}^{n}$ is pwd in $X$ with $\sum_{i=0}^{n} y_{i}=y$ and $0 \leq x_{i} \leq y_{i}$ for $1 \leq i \leq n$. Then

$$
\left[\sum_{i=1}^{n}\left\|T x_{i}\right\|_{Y}^{q}\right]^{1 / q} \leq\left[\sum_{i=0}^{n}\left\|T y_{i}\right\|_{Y}^{q}\right]^{1 / q}
$$

which implies $\|x\|_{Z} \leq\|y\|_{Z}$. This completes the proof.

REMARK. It is interesting to note that without the $\sigma$-completeness of $X,\|\cdot\|_{Z}$ defined in Corollary 3.4 is not in general a lattice norm. To see this let $i_{1}$ and $i_{2}$ be the inclusion maps $i_{1}: C[0,1] \rightarrow L_{q}[0,1]$ and $i_{2}: L_{q}[0,1] \rightarrow L_{r}[0,1], q<r$. Then $T=i_{2} i_{1}$ can be factored through $L_{q}$ which satisfies a lower $q$-estimate. However, if $0 \leq f \leq g$ in $C[0,1]$ are given by $g(t)=1$ for all $t$ in $[0,1]$ and $f$ is the piecewise linear function passing through the points $(0,1),(1 / 2-\varepsilon, 1),(1 / 2,0),(1 / 2+\varepsilon, 1)$, $(1,1)$, then $\|g\|_{Z}=1$ and $\|f\|_{Z} \geq\left[(1 / 2-\varepsilon)^{q / r}+(1 / 2-\varepsilon)^{q / r}\right]^{1 / q}=2^{1 / q}(1 / 2-\varepsilon)^{1 / r}$. Picking $\varepsilon$ small enough so that $2^{1 / q}(1 / 2-\varepsilon)^{1 / r}$ is $>1$ we get that $\|f\|_{Z}>1$ and $\|\cdot\|_{Z}$ is not monotone.

The following is a result of Conroy and Moore [3].

LEMma 3.5. Let $X$ be a Banach lattice. If $\left\{x_{i}^{*}\right\}_{i=1}^{n}$ is pwd in $X^{*}$ with $x_{i}^{*} \geq 0$, $x \geq 0$ in $X$, and $\varepsilon>0$ are given, there exists $\left\{x_{i}\right\}_{i=1}^{n}$ pwd in $X$ with $0 \leq x_{i} \leq x$, $\left(x_{i}, x_{i}^{*}\right)>\left(x, x_{i}^{*}\right)-\varepsilon$, and for $i \neq j,\left(x_{i}, x_{j}^{*}\right)<\varepsilon$.

PROOF. The proof is by induction on $n$. Letting $x_{1}=x$, the case $n=1$ is trivial. Let $n=2$. Since $0=\left(x, x_{1}^{*} \wedge x_{2}^{*}\right)=\inf \left\{\left(u, x_{1}^{*}\right)+\left(v, x_{2}^{*}\right): u+v=x, u \geq 0, v \geq 0\right\}$, there exist $w_{1} \geq 0$ and $w_{2} \geq 0$ in $X$ with $\left(w_{2}, x_{1}^{*}\right)+\left(w_{1}, x_{2}^{*}\right)<\varepsilon / 2$. Hence $\left(w_{2}, x_{1}^{*}\right)<\varepsilon / 2$ and $\left(w_{1}, x_{2}^{*}\right)<\varepsilon / 2$. Also $\left(w_{1}, x_{1}^{*}\right)>\left(x, x_{1}^{*}\right)-\varepsilon / 2$ and $\left(w_{2}, x_{2}^{*}\right)>$ $\left(x, x_{2}^{*}\right)-\varepsilon / 2$. Set $x_{1}=w_{1}-\left(w_{1} \wedge w_{2}\right)$ and $x_{2}=w_{2}-\left(w_{1} \wedge w_{2}\right)$. Then $x_{1} \wedge x_{2}=0$, $0 \leq x_{1} \leq x, 0 \leq x_{2} \leq x,\left(x_{1}, x_{2}^{*}\right) \leq\left(w_{1}, x_{2}^{*}\right)<\varepsilon / 2,\left(x_{2}, x_{1}^{*}\right) \leq\left(w_{2}, x_{1}^{*}\right)<\varepsilon / 2$, $\left(x_{1}, x_{1}^{*}\right)=\left(w_{1}, x_{1}^{*}\right)-\left(w_{1} \wedge w_{2}, x_{1}^{*}\right)>\left(x_{1}, x_{1}^{*}\right)-\varepsilon / 2-\left(w_{2}, x_{1}^{*}\right)>\left(x, x_{1}^{*}\right)-\varepsilon$ and similarly, $\left(x_{2}, x_{2}^{*}\right)>\left(x, x_{2}^{*}\right)-\varepsilon$.

Now suppose the result holds for $n=1,2, \ldots, k$ with $k \geq 2$ and consider the case of $k+1$ linear functionals. Apply the induction hypothesis to the $k$ linear functionals $x_{1}^{*}, x_{2}^{*}, \ldots, x_{k-1}^{*}, x_{k}^{*}+x_{k+1}^{*}$ and apply the case $k=2$ to the pair $x_{k}^{*}$ and $x_{k+1}^{*}$.

The following is a theorem of Tzafriri.

THEOREM 3.6. The following are equivalent for any Banach lattice $X$.

(i) No subspace of $X$ is isomorphic to $c_{0}$.

(ii) The canonical image of $X$ in $X^{* *}$ is a projection band.

PROOF [10, p. 34]. 
COROLLARY 3.7. Let $Y$ be a Banach lattice which contains no subspace isomorphic to $c_{0}$ and $T: X \rightarrow Y$ be a positive operator for some Banach lattice $X$. There exists a Banach lattice $Z$ which satisfies a lower q-estimate and positive operators $R: X \rightarrow Z$ and $S: Z \rightarrow Y$ with $T=S R$ if and only if there is a $K>0$ so that for all $\left\{x_{i}\right\}_{i=1}^{n}$ pwd in $X$

$$
\left[\sum_{i=1}^{n}\left\|T x_{i}\right\|_{Y}^{q}\right]^{1 / q} \leq K\left\|\sum_{i=1}^{n} x_{i}\right\|_{X} .
$$

Proof. (SUfFICIENCY) This is the special case of Theorem 2.1 with $x=$ $\sum_{i=1}^{n} x_{i}$.

(NECESSITY) First it is shown that $T^{*}$ satisfies the necessary condition of Theorem 2.2. Let $\varepsilon>0$ be given. Let $\left\{y_{i}^{*}\right\}_{i=1}^{n}$ be pwd in $Y^{*}$. We may assume, without loss of generality, that $y_{i}^{*} \geq 0$. Let $x \geq 0$ be in $X$ with $\|x\|_{X}=1$ and

$$
\left\|\sum_{i=1}^{n} T^{*} y_{i}^{*}\right\|_{X^{*}} \leq\left(x, \sum_{i=1}^{n} T^{*} y_{i}^{*}\right)+\varepsilon .
$$

Apply Lemma 3.5 to $\left\{T^{*} y_{i}^{*}\right\}_{i=1}^{n}$ using $\varepsilon / n$ in place of $\varepsilon$ to obtain $\left\{x_{i}\right\}_{i=1}^{n}$ pwd in $X$ with $0 \leq x_{i} \leq x$. Then using Hölder's inequality

$$
\begin{aligned}
\left\|\sum_{i=1}^{n} T^{*} y_{i}^{*}\right\|_{X^{*}} & \leq\left(x, \sum_{i=1}^{n} T^{*} y_{i}^{*}\right)+\varepsilon=\varepsilon+\sum_{i=1}^{n}\left(x, T^{*} y_{i}^{*}\right) \\
& \leq \varepsilon+\sum_{i=1}^{n}\left[\left(x_{i}, T^{*} y_{i}^{*}\right)+\varepsilon / n\right] \\
& =2 \varepsilon+\sum_{i=1}^{n}\left(T x_{i}, y_{i}^{*}\right) \leq 2 \varepsilon+\sum_{i=1}^{n}\left\|T x_{i}\right\|\left\|y_{i}^{*}\right\|_{Y^{*}} \\
& \leq 2 \varepsilon+\left[\sum_{i=1}^{n}\left\|T x_{i}\right\|_{Y}^{q}\right]^{1 / q}\left[\sum_{i=1}^{n}\left\|y_{i}^{*}\right\|_{Y^{*}}^{p}\right]^{1 / p} \\
& \leq 2 \varepsilon+K\left\|\sum_{i=1}^{n} x_{i}\right\|_{X}\left[\sum_{i=1}^{n}\left\|y_{i}^{*}\right\|_{Y^{*}}^{p}\right]^{1 / p} .
\end{aligned}
$$

Since $\varepsilon$ is arbitrary and $\sum_{i=1}^{n} x_{i} \leq x$ we get

$$
\left\|\sum_{i=1}^{n} T^{*} y_{i}^{*}\right\|_{X^{*}} \leq K\left[\sum_{i=1}^{n}\left\|y_{i}^{*}\right\|_{Y^{*}}^{p}\right]^{1 / p} \text { for all }\left\{y_{i}^{*}\right\}_{i=1}^{n} \operatorname{pwd} \text { in } Y^{*} .
$$

By Theorem 2.2 there exist a Banach lattice $W$ satisfying an upper $p$-estimate and positive operators $R: Y^{*} \rightarrow W$ and $S: W \rightarrow X^{*}$ with $T^{*}=S R$. Taking adjoints and using Theorem 3.1, $T^{* *}=R^{*} S^{*}$ with $W^{*}$ satisfying a lower $q$-estimate. Since $Y$ contains no subspace isomorphic to $c_{0}$. Theorem 3.6 gives that there is a positive projection $P: Y^{* *} \rightarrow Y$. Then restricting $T^{* *}$ to $X$ we get $T=T^{* *} P=R^{*} S^{*} P$ and $T$ factors through a Banach lattice satisfying a lower $q$-estimate. 
COROLlary 3.8. Let $X$ be a Banach lattice and $T: X \rightarrow Y$ be a positive operator for some Banach lattice $Y$. There is a Banach lattice $W$ that satisfies an upper p-estimate and a Banach lattice $Z$ that satisfies a lower q-estimate along with positive operators $R: X \rightarrow W, S: W \rightarrow Z$ and $Q: Z \rightarrow Y$ with $T=Q S R$ if and only if there is a $K>0$ so that for all $\left\{x_{i}\right\}_{i=1}^{n}$ and $\left\{w_{j}\right\}_{j=1}^{m}$ both pwd in $X$ with $\left|\sum_{i=1}^{n} x_{i}\right| \leq\left|\sum_{j=1}^{m} w_{j}\right|$, we have

$$
\begin{aligned}
& {\left[\sum_{i=1}^{n}\left\|T x_{i}\right\|_{Y}^{q}\right]^{1 / q} } \leq K\left[\sum_{j=1}^{m}\left\|w_{j}\right\|_{X}^{p}\right]^{1 / p} . \\
& X \stackrel{T}{\longrightarrow} Y \\
& R \downarrow \uparrow Q \\
& W \stackrel{S}{\longrightarrow} Z
\end{aligned}
$$

Proof. (SUfFiciency) Let $M$ be the upper $p$-estimate constant of $W$ and $N$ be the lower $q$-estimate constant of $Z$. Then if $\left\{x_{i}\right\}_{i=1}^{n}$ and $\left\{w_{j}\right\}_{j=1}^{m}$ are both pwd in $X$ with $\left|\sum_{i=1}^{n} x_{i}\right| \leq\left|\sum_{j=1}^{m} w_{i}\right|$ we have

$$
\begin{aligned}
{\left[\sum_{i=1}^{n}\left\|T x_{i}\right\|_{Y}^{q}\right]^{1 / q} } & =\left[\sum_{i=1}^{n}\left\|Q S R x_{i}\right\|_{Y}^{q}\right]^{1 / q} \leq\|Q\|\left[\sum_{i=1}^{n}\left\|S R x_{i}\right\|_{Z}^{q}\right]^{1 / q} \\
& \leq\|Q\| N\left\|\sum_{i=1}^{n} S R x_{i}\right\|_{Z} \leq\|Q\| N\|S\|\left\|\sum_{i=1}^{m} R w_{j}\right\|_{W} \\
& \leq\|Q\| N\|S\| M\left[\sum_{j=1}^{m}\left\|R w_{j}\right\|_{W}^{p}\right]^{1 / p} \\
& \leq\|Q\| N\|S\| M\|R\|\left[\sum_{j=1}^{m}\left\|w_{j}\right\|_{W}^{p}\right]^{1 / p}
\end{aligned}
$$

and the conclusion holds with $K=\|Q\| N\|S\| M\|R\|$.

(NECESSITY) By taking $\left\{w_{j}\right\}_{j=1}^{m}$ to be the singleton $\{x\}$, we get

$$
\left[\sum_{i=1}^{n}\left\|T x_{i}\right\|_{Y}^{q}\right]^{1 / q} \leq K\|x\|_{X}
$$

for all $\left\{x_{i}\right\}_{i=1}^{n}$ pwd in $X$ and all $x$ in $X$ with $\left|\sum_{i=1}^{n} x_{i}\right| \leq|x|$. Applying Theorem 2.1 we obtain a Banach lattice $Z$ which satsfies a lower $q$-estimate and positive operators $V: X \rightarrow Z$ and $Q: Z \rightarrow Y$ with $T=Q V$.

Now let $\left\{x_{i}\right\}_{i=1}^{n}$ be pwd in $X$ and let $\varepsilon>0$ be given. Let $\left\{w_{j}\right\}_{j=1}^{m}$ be pwd in $X$ so that $\left|\sum_{j=1}^{m} w_{j}\right| \leq\left|\sum_{i=1}^{n} x_{i}\right|$ and

$$
\left\|\sum_{i=1}^{n} V x_{i}\right\|_{Z} \leq(1+\varepsilon)\left[\sum_{j=1}^{m}\left\|V w_{j}\right\|_{Y}^{q}\right]^{1 / q} .
$$


Since $V x=T x$ for $x$ in $X$

$$
\begin{aligned}
\left\|\sum_{i=1}^{n} V x_{i}\right\|_{Z} & \leq(1+\varepsilon)\left[\sum_{j=1}^{m}\left\|T w_{j}\right\|_{Y}^{q}\right]^{1 / q} \\
& \leq(1+\varepsilon) K\left[\sum_{i=1}^{n}\left\|x_{i}\right\|_{X}^{p}\right]^{1 / p} .
\end{aligned}
$$

Since $\varepsilon>0$ is arbitrary, $V$ satisfies the sufficient condition of Theorem 2.2. We obtain a Banach lattice $W$ which satisfies an upper $p$-estimate and positive operators $R: X \rightarrow W$ and $S: W \rightarrow Z$ with $V=S R$. Hence $T=Q S R$ and the proof is complete.

REMARK. In a sense, Corollary 3.8 is a dead end. That is, $W$ and $Z$ come in the wrong order in the factorization, so that these techniques do not imply that such a $T$ can be factored through a uniformly convex lattice. Corollary 3.12 will show that reversing the order of $W$ and $Z$ does give the desired result. However, Corollary 3.9 shows that the hypothesis of Corollary 3.8 is required for a factorization through a uniformly convex lattice. We do not know if this hypothesis is also sufficient, but it is not sufficient using these techniques.

COROLlARY 3.9. If $T: X \rightarrow Y$ is a positive operator that can be factored through a super-reflexive Banach lattice $Z$ with positive operators $R: X \rightarrow Z$ and $S: Z \rightarrow Y$, then there are positive constants $p, q$ and $K$ so that for all $\left\{x_{i}\right\}_{i=1}^{n}$ and $\left\{w_{j}\right\}_{j=1}^{m}$ both $p w d$ in $X$ with $\left|\sum_{i=1}^{n} x_{i}\right| \leq\left|\sum_{j=1}^{m} w_{j}\right|$ we have

$$
\left[\sum_{i=1}^{n}\left\|T x_{i}\right\|_{Y}^{q}\right]^{1 / q} \leq K\left[\sum_{j=1}^{m}\left\|w_{j}\right\|_{X}^{p}\right]^{1 / p} .
$$

ProOF. There exist positive constants $p$ and $q$ so that $Z$ satisfies an upper $p$-estimate and a lower $q$-estimate. Now apply Corollary 3.8 .

The following inequality is due to Jessen.

LEMMA 3.10. If $1<p<q$

$$
\left[\sum_{j=1}^{n}\left[\sum_{i=1}^{m}\left|a_{i j}\right|^{p}\right]^{q / p}\right]^{1 / q} \leq\left[\sum_{i=1}^{m}\left[\sum_{j=1}^{n}\left|a_{i j}\right|^{q}\right]^{p / q}\right]^{1 / p}
$$

Proof [6, p. 31].

We now prove a technical lemma to facilitate the proof of Corollary 3.12.

LEMMA 3.11. Suppose $1<p<q$ and the Banach lattice $W$ satisfies an upper p-estimate. Let $Z_{0}=\left\{w \in W:\|w\|_{Z}<\infty\right\}$ where $\|\cdot\|_{Z}$ is defined on $W$ by

$$
\|w\|_{Z}=\sup \left\{\left[\sum_{i=1}^{n}\left\|w_{i}\right\|_{W}^{q}\right]^{1 / q}\right\}
$$

the sup being taken over all $\left\{w_{i}\right\}_{i=1}^{n}$ pwd in $W$ with $\left|\sum_{i=1}^{n} w_{i}\right| \leq|w|$. Let $Z$ be the $\|\cdot\|_{Z}$-completion of $Z_{0}$.

Then $Z$ satisfies an upper p-estimate. 
PROOF. Let $\varepsilon>0$ be given and let $M$ be the upper $p$-estimate constant of $W$. If $\left\{z_{i}\right\}_{i=1}^{n}$ is pwd in $Z_{0}$, pick $\left\{w_{j}\right\}_{j=1}^{m}$ pwd in $W$ so that $\left|\sum_{j=1}^{m} w_{j}\right| \leq\left|\sum_{i=1}^{n} z_{i}\right|$ and

$$
\left\|\sum_{i=1}^{n} z_{i}\right\|_{Z} \leq(1+\varepsilon)\left[\sum_{j=1}^{m}\left\|w_{j}\right\|_{W}^{q}\right]^{1 / q} .
$$

For each $j$ pick, using the decomposition property, $\left\{w_{i j}\right\}_{i=1}^{n}$ pwd in $W$ so that $\left|w_{i j}\right| \leq\left|z_{i}\right|$ and $\sum_{i=1}^{n} w_{i j}=w_{j}$. Using Lemma 3.10

$$
\begin{aligned}
\left\|\sum_{i=1}^{n} z_{i}\right\|_{Z} & \leq(1+\varepsilon)\left[\sum_{j=1}^{m}\left\|w_{j}\right\|_{W}^{q}\right]^{1 / q} \\
& \leq(1+\varepsilon) M\left[\sum_{j=1}^{m}\left[\sum_{i=1}^{n}\left\|w_{i j}\right\|_{W}^{p}\right]^{q / p}\right]^{1 / q} \\
& \leq(1+\varepsilon) M\left[\sum_{i=1}^{n}\left[\sum_{j=1}^{m}\left\|w_{i j}\right\|_{W}^{q}\right]^{p / q}\right]^{1 / p} \\
& \leq(1+\varepsilon) M\left[\sum_{i=1}^{n}\left\|z_{i}\right\|_{Z}^{p}\right]^{1 / p}
\end{aligned}
$$

and $Z_{0}$ satisfies an upper $p$-estimate with estimate constant $M$. Using Proposition 1.6, $Z$ also satisfies an upper $p$-estimate with estimate constant $M$.

COROLlaRY 3.12. Let $X$ be a Banach lattice and $T: X \rightarrow Y$ be a positive operator for some Banach lattice $Y$. Then $T$ can be factored through a superreflexive Banach lattice if and only if there are constants $p$ and $q$ and a Banach lattice $Z$ which satisfies a lower q-estimate and a Banach lattice $W$ which satisfies an upper p-estimate and positive operators $R: X \rightarrow Z, S: Z \rightarrow W$, and $Q: W \rightarrow Y$ with $T=Q S R$.

ProOf. (SUFFICIENCY) This is clearly true since a super-reflexive Banach lattice satisfies both an upper and a lower estimate.

(NECESSITY) Since $Z$ satisfies a lower $q$-estimate, $S$ satisfies the necessary condition of Theorem 2.1. The construction in the proof of Theorem 2.1 yields a Banach lattice $V$ which satisfies a lower $q$-estimate through which $S$ can be factored. Lemma 3.11 states that the upper $p$-estimate of $W$ is preserved under this construction and hence $V$ is super-reflexive.

DEFINITION. Let $\varepsilon$ be given with $0<\varepsilon \leq 2$. We say that two points $x_{1}, x_{2} \in X$ form a $(1, \varepsilon)$-branch in $X$ if $\left\|x_{1}-x_{2}\right\| \geq \varepsilon$. Assume that a $(n-1, \varepsilon)$-branch $(n \geq 2)$ has been defined. We shall say that the $2^{n}$ points $x_{1}, x_{2}, \ldots, x_{2^{n}}$ form a $(n, \varepsilon)$-branch in $X$ if

(a) $\left\|x_{2 i}-x_{2 i-1}\right\| \geq \varepsilon$ for $i=1,2, \ldots, 2^{n-1}$ and 
(b) the midpoints $\left(x_{2 i}+x_{2 i-1}\right) / 2, i=1,2, \ldots, 2^{n-1}$, form a $(n-1, \varepsilon)$-branch. We say that $X$ has the Finite Tree Property if, for some $\varepsilon, 0<\varepsilon \leq 2$, one can find, for every $n \geq 1$, a $(n, \varepsilon)$-branch contained in the unit ball of $X$.

THEOREM 3.13 (JAMES). A Banach space is super-reflexive if and only if it does not have the Finite Tree Property.

PROOF. [2, p. 231].

DEFINITION. Let $X$ and $Y$ be Banach spaces with bases $\left\{x_{i}\right\}_{i=1}^{\infty}$ and $\left\{y_{i}\right\}_{i=1}^{\infty}$, respectively. The operator $T_{\lambda}: X \rightarrow Y$ is said to be a diagonal operator if

$$
T_{\lambda}\left(\sum_{i=1}^{\infty} a_{i} x_{i}\right)=\sum_{i=1}^{\infty} \lambda_{i} a_{i} y_{i}
$$

for some sequence of scalars $\left\{\lambda_{i}\right\}_{i=1}^{\infty}$.

LEMMA 3.14 (CROFT). Let $T_{\lambda}: X \rightarrow Y$ be a diagonal operator. If $\| T_{\lambda}^{n}-$ $T_{\lambda} \| \stackrel{n}{\rightarrow} 0$ where $T_{g}^{n}: X \rightarrow Y$ is defined by $T_{\lambda}^{n}\left(\sum_{i=1}^{\infty} a_{i} x_{i}\right)=\sum_{i=1}^{n} \lambda_{i} a_{i} y_{i}$, then $T_{\lambda}$ is compact.

PROOF. [14, p. 285].

LEMMA 3.15. Let $T_{\lambda}: c_{0} \rightarrow c_{0}$ be the diagonal operator defined by $T_{\lambda}\left(\sum_{i=1}^{\infty} a_{i} e_{i}\right)$ $=\sum_{i=1}^{\infty} \lambda_{i} a_{i} e_{i}$ where $\left\{e_{i}\right\}_{i=1}^{\infty}$ is the usual basis of $c_{0}$. If $T_{\lambda}$ can be factored through a Banach lattice which satisfies a lower q-estimate, then $\left\{\lambda_{i}\right\}_{i=1}^{\infty} \in l_{q}$.

PROOF. Using Theorem 2.1, there is a $K>0$ so that

$$
\left[\sum_{i=1}^{n}\left|\lambda_{i}\right|^{q}\right]^{1 / q}=\left[\sum_{i=1}^{n}\left\|\lambda_{i} e_{i}\right\|^{q}\right]^{1 / q} \leq K\left\|\sum_{i=1}^{n} e_{i}\right\|=K
$$

and $\left\{\lambda_{i}\right\}_{i=1}^{\infty} \in l_{q}$.

DEFINITION (BEAUZAMY). An operator $T: X \rightarrow Y$ is said to have the Finite Tree Property if, for some $\varepsilon, 0<\varepsilon \leq 2$, for all $n \geq 1$, one can find in the unit ball of $X, 2^{n}$ points $x_{1}, x_{2}, \ldots, x_{2^{n}}$ so that the images $T x_{1}, T x_{2}, \ldots, T x_{2^{n}}$ form a $(n, \varepsilon)$-branch in $Y$. An operator $T$ is called a uniformly convexifying operator if $T$ does not have the Finite Tree Property.

Note that the identity operator on a uniformly convex space is uniformly convexifying. Beauzamy noted in $[\mathbf{1}]$ that a compact operator is necessarily uniformly convexifying.

EXAMPLE. The following is an example of a uniformly convexifying operator that factors through no uniformly convex Banach lattice.

Let $T_{\lambda}: c_{0} \rightarrow c_{0}$ be the diagonal operator with $\lambda_{i}=1 / \log (i+1)$. Using Lemma 3.14

$$
\left\|\sum_{i=n+1}^{\infty} \lambda_{i} a_{i} e_{i}\right\| \leq \sup _{i \geq n+1}\left|\lambda_{i}\right|\left\|\sum_{i=n+1}^{\infty} a_{i} e_{i}\right\| \stackrel{n}{\rightarrow} 0,
$$

and $T_{\lambda}$ is compact and hence uniformly convexifying. However, since $\left\{\lambda_{i}\right\}_{i=1}^{\infty}$ belongs to no $l_{q}$, Lemma 3.15 states that $T_{\lambda}$ does not factor through any uniformly convex Banach lattice.

REMARK. Beauzamy knew that a uniformly convexifying operator need not factor through a uniformly convex space. Hence his choice of terminology could be 
open to question. A better choice might be super-reflexive operator, since "nonreflexive operators" are not "finitely representable" in such an operator.

Although super-reflexive and isomorphic to a uniformly convex space are equivalent for Banach spaces, the question becomes more complex for operators (even positive ones).

\section{REFERENCES}

1. B. Beauzamy, Opèrateurs uniformèment convexifiants, Studia Math. 57 (1976), 103-139.

2. - Introduction to Banach spaces and their geometry, North-Holland, Amsterdam, 1982.

3. J. L. Conroy and L. C. Moore, Local reflexivity in Banach lattices (preprint).

4. W. J. Davis, T. Figiel, W. B. Johnson and A. Pełczyński, Factoring weakly compact operators, J. Funct. Anal. 17 (1974), 311-327.

5. M. M. Day, Normed linear spaces, 3rd ed., Springer-Verlag, New York, 1973.

6. N. Dunford and J. Schwartz, Linear operators, Part 1: General theory, Interscience, New York, 1958.

7. G. H. Hardy, J. E. Littlewood and G. Polya, Inequalities, Cambridge Univ. Press, 1952.

8. R. C. James, Super-reflexive spaces with bases, Pacific J. Math. 41 (1972), 409-419.

9. J. Lindenstrauss and L. Tzafriri, Classical Banach spaces. I, Sequence spaces, Springer-Verlag, New York, 1977.

10. _ Classical Banach spaces. II, Function spaces, Springer-Verlag, New York, 1979.

11. W. A. J. Luxemburg and A. C. Zaanen, Riesz spaces, Amsterdam, North-Holland, 1971.

12. A. Pełczyński, Some problems on bases in Banach and Fréchet spaces, Israel J. Math. 2 (1964), 132-138.

13. I. Singer, Bases in Banach spaces. I, Springer-Verlag, New York, 1970.

14. A. E. Taylor, Introduction to functional analysis, Wiley, New York, 1958.

15. B. Z. Vulikh, Introduction to the theory of partially ordered spaces, Wolters-Noordhoff, Groningen, 1967.

Department of Mathematics, University of Tennessee, Chattanooga, TenNESSEE 37403 